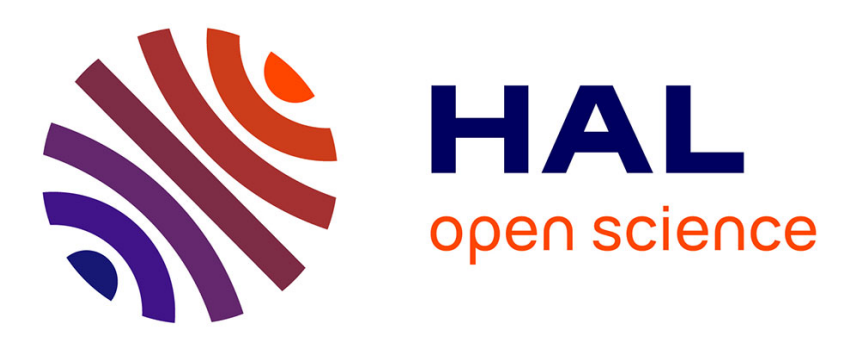

\title{
Supercritical Eckhaus Instability for Surface-Tension-Driven Hydrothermal Waves
}

Nathalie Mukolobwiez, Arnaud Chiffaudel, François Daviaud

\section{To cite this version:}

Nathalie Mukolobwiez, Arnaud Chiffaudel, François Daviaud. Supercritical Eckhaus Instability for Surface-Tension-Driven Hydrothermal Waves. Physical Review Letters, 1998, 80, pp.4661-4664. 10.1103/PhysRevLett.80.4661 . cea-01373936

\section{HAL Id: cea-01373936 https://hal-cea.archives-ouvertes.fr/cea-01373936}

Submitted on 29 Sep 2016

HAL is a multi-disciplinary open access archive for the deposit and dissemination of scientific research documents, whether they are published or not. The documents may come from teaching and research institutions in France or abroad, or from public or private research centers.
L'archive ouverte pluridisciplinaire HAL, est destinée au dépôt et à la diffusion de documents scientifiques de niveau recherche, publiés ou non, émanant des établissements d'enseignement et de recherche français ou étrangers, des laboratoires publics ou privés. 


\title{
Supercritical Eckhaus Instability for Surface-Tension-Driven Hydrothermal Waves
}

\author{
Nathalie Mukolobwiez, ${ }^{1}$ Arnaud Chiffaudel, ${ }^{1,2}$ and François Daviaud ${ }^{1}$ \\ ${ }^{1}$ Service de Physique de l'État Condensé, CEA Saclay, 91191 Gif-sur-Yvette Cedex, France \\ ${ }^{2}$ Laboratoire de Physique Statistique, E.N.S., 24 rue Lhomond, 75231 Paris Cedex 05, France
}

(Received 3 July 1997)

\begin{abstract}
We study the nonlinear dynamics of hydrothermal waves produced by a surface-tension-driven convective flow in a long and thin annular channel heated from the side. Above onset, the supercritical traveling wave pattern undergoes a secondary instability: a supercritical Eckhaus instability. This leads to a small-wave-number phase-modulated nonlinear mode, and shows the first experimental evidence of a nonlinearly saturated phase instability mode for traveling wave patterns. At higher forcing level, this secondary pattern is subject to a tertiary instability. This mode is an amplitude mode characterized by traveling hole patterns, i.e., space-time defects that change the wave number. [S0031-9007(98)06211-5]
\end{abstract}

PACS numbers: 47.20. $-\mathrm{k}, 47.27 .-\mathrm{i}, 47.35 .+\mathrm{i}, 47.54 .+\mathrm{r}$

Understanding the transition from order to spatiotemporal chaos in extended nonlinear systems remains an important problem. Physicists first started to study systems unstable to stationary spatial patterns such as, e.g., Rayleigh-Bénard convection [1] and, later on, oscillatory instabilities and nonlinear traveling-wave patterns. For 1D waves, two closely related mechanisms - Eckhaus [2] and Benjamin-Feir (BF) [3] - could be invoked. They have been intensively searched and studied in several physical systems [4-8]. This research has also been strongly stimulated by numerical experiments on the complex Ginzburg-Landau equation (CGLE) $[9,10]$. Remarkable predictions concern the existence of nonlinear phase regimes, presumably only in a tiny region close to the BF stability limit of the CGLE phase diagram: In this region, $\mathrm{BF}$ unstable patterns may show phase turbulence $[9,10]$, whereas the Eckhaus unstable pattern could bifurcate on a supercritical way $[4,11]$ leading to nonlinearly saturated phase modulations. In this Letter, an annular wave pattern is produced by a supercritical oscillatory instability. It behaves as a 1D traveling wave with periodic boundary conditions and should be described by a cubic CGLE. At higher order parameter, this pattern undergoes a nonlinearly saturated phase instability - a supercritical Eckhaus instability - in the form of a persistent smallwave-number traveling modulation. This is, as far as we know, the first report of a nonlinear phase pattern on the way to spatiotemporal chaos for a large system, and confirms the above predictions.

Experimental setup.-An annular convection channel (Fig. 1) with a glass bottom and vertical copper walls (cf. Ref. [12] for rectangular geometry) is filled with a thin layer of silicon oil of viscosity $\nu=0.65 \mathrm{cS}$ and Prandtl number $P=10$. The fluid surface is free. The channel is $1 \mathrm{~cm}$ wide and its mean radius is $L=$ $8 \mathrm{~cm}$. The perimeter is $50 \mathrm{~cm}$ which corresponds to an aspect ratio $\Gamma=2 \pi L / h=300$ for $h=1.7 \mathrm{~mm}$. The outer copper wall is cooled by a thermoregulated fluid circulation at $293 \mathrm{~K}$ while the inner copper block is heated electrically. Thermocouples allow accurate measurements of the temperature difference $\Delta T$ across the channel. This difference is typically established with a $\pm 20 \mathrm{mK}$ stability. Convective patterns are observed through the glass bottom by shadowgraphy. Images are digitized and processed numerically as spatiotemporal diagrams of 512 data points extracted from a mean circle and plotted along time.

Basic flow. - The convective flow created by this horizontal thermal gradient was described by Daviaud and Vince [12]. The thermal gradient across the cell induces a surface tension gradient on the free surface of the fluid. Because of the Marangoni effect, this gradient generates a surface flow towards the cold side, with a bottom recirculation: The basic flow is a long annular roll. Above a given threshold $\Delta T_{0}$, and for low fluid thickness $h<$ $2.8 \mathrm{~mm}$, this flow becomes unstable with respect to horizontal traveling waves (TW) which propagate along the channel (Fig. 1), i.e., the roll axis. This behavior characterizing hydrothermal waves, was predicted by Smith and Davis [13], detected in several experiments [12,14], and recently studied numerically in conditions close to our experimental situation by Mercier and Normand [15].

In this Letter, we report the observation of hydrothermal traveling waves near the minimum of the

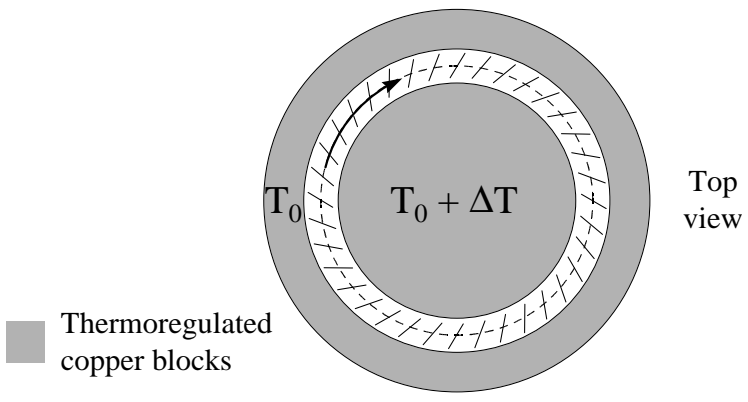

FIG. 1. Schematics of the annular channel with sketch of traveling hydrothermal waves and acquisition circle (- - -). 
stability curve (see Fig. 3 of Ref. [12]), i.e., for $h=(1.7 \pm 0.1) \mathrm{mm}$. At this point, the first bifurcation to hydrothermal waves occurs at $\Delta T_{0}=3.5 \mathrm{~K}$. This value is locally almost independent of $h$. This stability of the onset simplifies the experimental task because the fluid height decreases typically by $0.01 \mathrm{~mm} \times h^{-1}$, due to evaporation and capillarity. We note also that the thickness of the fluid layers varies along the channel owing to a slight nonhorizontality of the cell and even more negligible curvature of the bottom plate. For the present set of experiments, $h$ was kept in a $\pm 0.04 \mathrm{~mm}$ range along the channel. This small imperfection induces a small (typically $4 \%$ ) modulation of the local wave number of the pattern around its mean value. This modulation can be easily subtracted during the data processing phase.

Just above onset $\Delta T_{0}$, the waves invade the cell leading generally, as reported for the rectangular cell, to a classical source/sink pattern. However, after a relatively long transient, the defects generally annihilate, leading to a single homogeneous plane wave rotating along the annulus in either direction. The wave number selected by the source for a source/sink system is typically $k_{0}=61 / L$, whereas defectless wave patterns select between 50 and 57 wavelengths in the cell. This first bifurcation is supercritical, as shown in Fig. 2: The amplitude of the wave pattern behaves like $\left(\Delta T-\Delta T_{0}\right)^{1 / 2}$. Thus this system is a very good prototype for the study of supercritical homogeneous traveling wave patterns with periodic boundary conditions in a large aspect ratio container. It should be compared to binary-fluid convection for a subcritical TW bifurcation [5,6], to oscillatory instability studies in argon with small aspect ratio [4] or nonhomogeneous patterns [16], and to oscillatory rotating convection [7].

Notation and data processing.-Following the usual framework of nonlinear patterns, we will try to extract

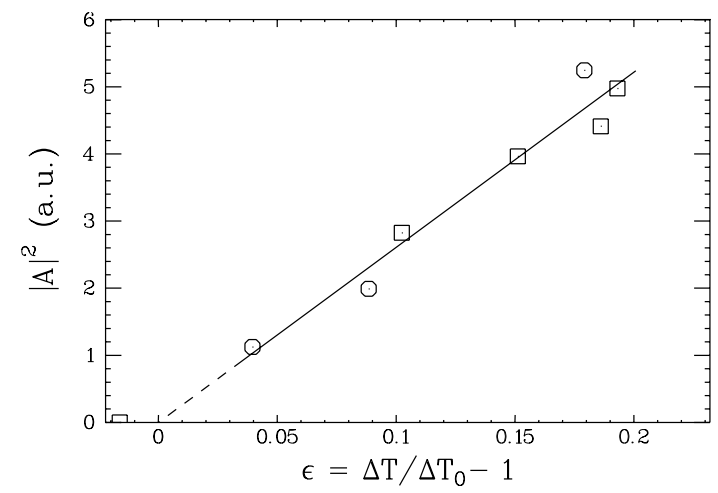

FIG. 2. Critical behavior of the first instability of the channel flow: the onset of traveling waves. We have plotted the squared amplitude $|A|^{2}$ for various values of the control parameter $\Delta T$, and also various wave patterns. Squares correspond to homogeneous left or right TW, whereas circles represents source/sink patterns. Data for different wave-number patterns are collected together and are coherent with threshold $\Delta T_{0}=$ $3.50 \mathrm{~K}$ and critical exponent $1 / 2$. from the spatiotemporal images the quantities which could be directly written in a nonlinear model equation such as CGLE. For that purpose, the real shadowgraphic data $\theta_{r}(x, t)$ related to the thermal field can be written as

$$
\begin{gathered}
\theta_{r}(x, t)=\theta_{c}(x, t)+\text { c.c }+\ldots, \\
\theta_{c}(x, t)=A(X, T) \exp \left[i\left(\omega_{0} t-k_{0} x\right)\right], \\
A(X, T)=R(X, T) \exp [i \Phi(X, T)],
\end{gathered}
$$

where $\theta_{c}$ is called the complexified signal, $A(X, T)$ is a complex amplitude depending on the slow variables $X$ and $T, R(X, T)$ is its modulus, c.c. stands for the complex conjugate, and the dots for harmonics. In order to be demodulated, real data are complexified by the three following operations [17]: a Fourier transform of $\theta_{r}$, a wide bandpass filtering around the positive fundamental in Fourier space, and then the reverse Fourier transform which creates the complexified signal $\theta_{c}$. The complex spatiotemporal image of $\theta_{c}$ will be viewed as follows:

(i) Its modulus. $-R(X, T)$, i.e., the local amplitude of the wave.

(ii) The two gradients of its phase $\varphi \cdot-\partial_{t} \varphi=\omega_{0}+$ $\partial_{T} \Phi(X, T)$ and $\partial_{x} \varphi=k_{0}+\partial_{X} \Phi(X, T)$, i.e., the local frequency and local wave number of the pattern.

Results. - The first perturbations of the TW field observed above $\Delta T_{0}$ consists of the appearance of small phase modulations of the pattern. The system appears to be very sensitive to such modulations: Once $\epsilon=$ $\Delta T / \Delta T_{0}-1$ is positive, due to noise, we observe very small nonhomogeneities of the local wave number traveling slowly along the cell. The velocity of these structures is typically 6 times slower than the phase velocity. Their amplitude is close to our noise detection level, i.e., of order $0.1 / L$ for the wave-number modulation $\partial_{X} \Phi$. To get more information we forced these modulations by dropping a few drops of oil into the channel. This perturbation is done extremely fast with respect to the thermal time.

The relaxation of this forced perturbation is illustrated in Fig. 3 which shows the local wave number $\partial_{X} \Phi$ along time for the first $2000 \mathrm{~s}$. After two rounds, the spatial harmonics of the initial pulse-shaped pattern have relaxed. The remaining pattern is a sine modulation at the size of the box. This corresponds to a compression-dilatation mode of the wavelength of the basic TW mode, which will be called carrier, by analogy with the radio-frequency domain. However, this phase modulation is also slowly damped. To measure this damping rate, we demodulate this $\partial_{X} \Phi$ signal. After a spatial bandpass filtering at about $K_{M}=1 / L$ - the size of the box - we extract the amplitude $q(T)$ of the local wave-number modulation:

$$
\partial_{X} \Phi(X, T)=q(T) \exp \left[i\left(\Omega_{M} T-K_{M} X\right)\right]+\ldots
$$

This amplitude is plotted in Fig. 4. It is exponentially damped with a characteristic time $\tau=4500 \mathrm{~s}$. This explains why the very small spontaneous modulations, 


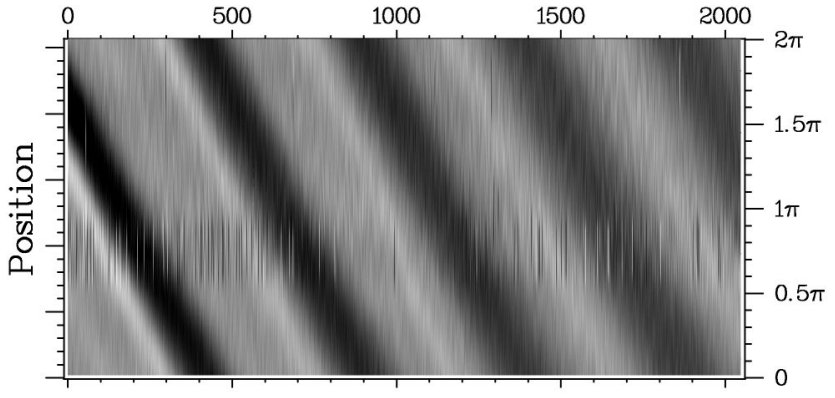

Time (s)

FIG. 3. Evolution of the perturbation (just before $t=0$ ) of a stable TW pattern for $\Delta T=4.0 \mathrm{~K}$ or $\epsilon=0.15$. The carrier TW pattern consists of 55 wavelengths $\left(k_{0}=55 / L\right)$ traveling at frequency $\omega_{0} / 2 \pi=0.51 \mathrm{~Hz}$. This space-time picture with periodic spatial boundary conditions represents the local wave number $\partial_{X} \Phi$ of the pattern: dark (clear) regions show low (high) wave numbers, i.e., dilatations (compressions) of the TW carrier. For noise reduction this image has been filtered in space: It shows only the first four modes. The period of the first mode, at wave number $K_{M}=1 / L$, is $\tau_{M}=2 \pi / \Omega_{M}=$ 485 s, i.e., typically 300 times the period of the carrier.

due to experimental noise, are regularly observed over several hundred seconds. In fact, this very low rate is the signature of a secondary bifurcation for slightly higher temperature gradient.

Once $\epsilon$ is set greater than $\epsilon_{1}=0.66$ we observe the wave-number modulation at $K_{M}=1 / L$ to globally grow, saturate and persist for several hours (Fig. 5). This transition has no hysteresis. It's a supercritical Hopf bifurcation. The bifurcated modulation amplitude is quite small compared to the forced modulation: typically $2-$ 3 times the noise level. This secondary instability is a phase instability because the local amplitude $R$ of the carrier wave is almost unaffected by the modulation. It is also an Eckhaus instability because it affects the

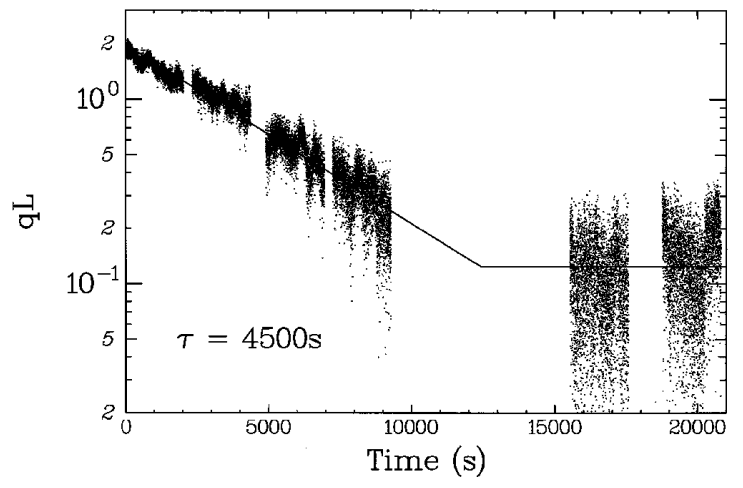

FIG. 4. Amplitude $q(T)$ of the wave-number modulation for its first mode-of the size of the box-for $20000 \mathrm{~s}$ after perturbation of the carrier TW. The noise level $0.12 / L$ is achieved after $12000 \mathrm{~s}$, for this very strong perturbation of order $2 / L$, i.e., $4 \%$ of the mean wave number $k_{0}=55 / L$. The first 2048 s corresponds to the data of Fig. 3. longitudinal variable. In fact, by increasing the constraint above $\epsilon_{2}=0.9$ we recover the usual Eckhaus behavior.

Figure 6 represents the evolution of a $k_{0}=52 / L$ modulated carrier pattern when $\epsilon$ is increased above $\epsilon_{2}$. We observe the spontaneous growth of high wavenumber modulations leading to space-time defects or amplitude holes [16], where the amplitude goes to zero and the phase jumps by $\pi$ (see caption for details). After six phase jumps, the mean wave number of the carrier TW has decreased to $k_{0}=46 / L$ and the patterns relax towards a nonmodulated state. Note that this example corresponds to a strong increase of the control parameter $\epsilon$. For smaller steps above $\epsilon_{2}$, the transient state lasts much longer: Many more traveling amplitude holes are observed, whereas only five or six of them reach the zero-amplitude level and change the carrier pattern wave number. This recalls the slow chaotic state [5] for the Eckhaus transition of subcritical TW. Further increase of the thermal constraint produces a new Eckhaus modulation followed again by a loss of one or two wavelengths. The scenario reproduces itself several times until, for $k_{0}$ of order $40 / L$, weak turbulence arises, consisting mainly of amplitude hole defects.

The Eckhaus instability has been carefully studied for TW patterns in Rayleigh-Bénard convection [4-7] or wakes of bluff bodies [8]. In those experiments, this instability always appears to be subcritical, even if not explicitly stated, with exponential growth of phase modulations up to the point where an amplitude hole breaks the underlying carrier TW. No stable nonlinear phase mode was ever reported. In the present experiment, the Eckhaus instability is encountered for increasing control parameter which means that the stability domain, unlike the classical parabola for Rayleigh-Bénard convection, is somewhat slanted. Nevertheless, the existence of stable nonlinear solutions for the phase equation - the modulated pattern - has been predicted analytically by Fauve

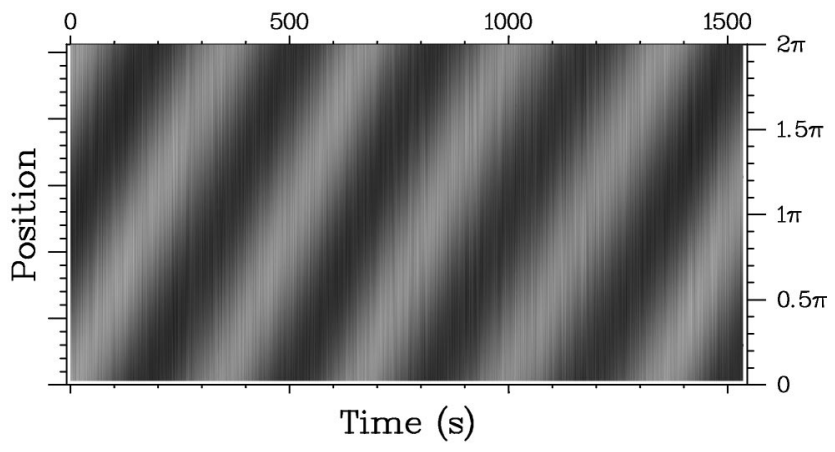

FIG. 5. Space-time image of the local wave number of the $k_{0} L=51$ and $\omega_{0} / 2 \pi=0.61 \mathrm{~Hz}$ hydrothermal wave for $\Delta T=6.6 \mathrm{~K}$ or $\epsilon=0.88$. This wave-number modulation is nonlinearly saturated and propagates steadily along time. According to Eq. (4), we measure $q L=0.20 \pm 0.04,2 \pi / \Omega_{M}=$ $332 \mathrm{~s}$, and $K_{M} L=1$. Higher spatial frequencies are filtered and the contrast is enhanced 8 times with respect to Fig. 3. 


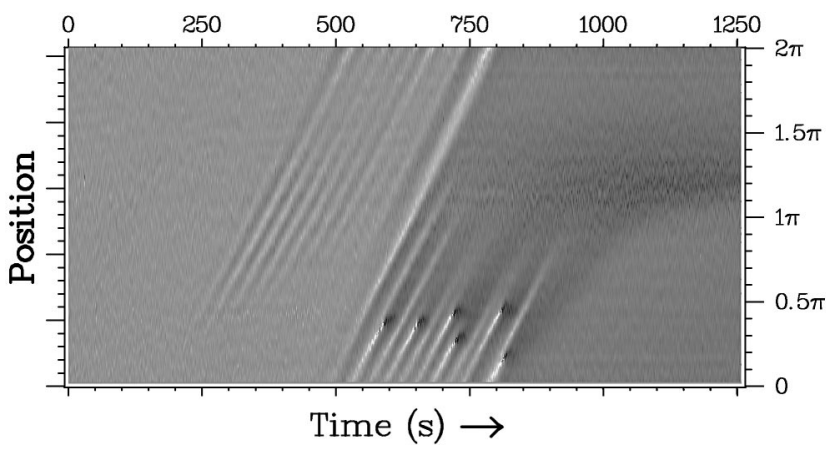

FIG. 6. Transient evolution of a $k_{0}=52 / L$ Eckhaus modulated TW pattern toward a stable $k_{0}=46 / L$ pattern, when $\epsilon$ grows from 0.69 to 0.99 above $\epsilon_{2}$. This image represents the local wave number without any filtering or enhancement: The $K_{M}=1 / L$ modulation of the initial pattern is thus not visible. Suddenly, near $t=250 \mathrm{~s}$, higher spatial frequencies start growing and generate six traveling wave-number peaks. The velocity of these structures is the same as the phase velocity of the previous modulation. The wave-number peaks correspond to growing phase jumps: once they reach $\pi$, with the local amplitude simultaneously attaining zero (not shown) and the local wave number changing sign, they create six spacetime defects which each annihilate one wavelength of the TW pattern.

[11] and calculated by Janiaud et al. [4]. The latter also observed supercritical pulses in numerical CGLE in the vicinity of the $\mathrm{BF}$ instability curve, a feature which is very similar to the one reported here. We did not try to measure the CGLE coefficients relevant to our experiment but, owing to the very long relaxation time observed just above the first bifurcation threshold (Fig. 4), we estimate the BF line to be close. Finally, note that our observations are limited to a small window in the $\Delta T$ range: Once the phase modulation amplitude grows, it triggers an amplitude instability and its train of correlated spacetime defects. This behaves like the growing long-lived transients for subcritical Eckhaus [4-8], where the $K_{M}=$ $1 / L$ mode grows until a defect is created. However, in our case, the defects are nucleated by the growth of higher wave-number modes, e.g., harmonics number 6 or 7 . We may thus suspect that, whereas the low wave-number modes are nonlinearly damped, the spatial harmonics which arise further above Eckhaus onset, are not. These defects are traveling hole patterns whose evolution is qualitatively similar to the Nozaki-Bekki hole solution of CGLE [16,18] or homoclinic holes [19]. These holes precede "amplitude" or "defect" turbulence for higher $\Delta T$, which is currently being studied.

In conclusion, we wish to emphasize the qualitative similarity of this experimental observation with numerical studies of the phase diagram for BF unstable regimes of supercritical CGLE (Fig. 3 of Ref. [9]). As CGLE presents a continuous transition from pure waves to phase turbulence and then to defect turbulence, our experiment transits from pure waves to nonlinearly saturated modulation-a pure nonlinear phase mode-and then to an amplitude regime consisting of space-time defects.

We wish to thank S. Fauve, B. Janiaud, J. Lega, and O. Dauchot for fruitful discussions, and C. Gasquet for technical assistance. Laboratoire de Physique Statistique is Laboratoire associé to the Centre National de la Recherche Scientifique.

[1] F. Daviaud, M. Bonetti, and M. Dubois, Phys. Rev. A 42, 3388 (1990).

[2] W. Eckhaus, Studies in Nonlinear Stability Theory (Springer, New York, 1965).

[3] T.B. Benjamin and J.E. Feir, J. Fluid Mech. 27, 417 (1967).

[4] B. Janiaud, A. Pumir, D. Bensimon, V. Croquette, H. Richter, and L. Kramer, Physica (Amsterdam) 55D, 269 (1992).

[5] P. Kolodner, Phys. Rev. A 46, 6431 (1992).

[6] G. W. Baxter, K. D. Eaton, and C. M. Surko, Phys. Rev. A 46, R1735 (1992).

[7] Y. Liu and R. E. Ecke, Phys. Rev. Lett. 78, 4391 (1997).

[8] T. Leweke and M. Provansal, J. Fluid Mech. 288, 265 (1995).

[9] B.I. Shraiman et al., Physica (Amsterdam) 57D, 241 (1992).

[10] H. Chaté, Nonlinearity 7, 185 (1994).

[11] S. Fauve, in Instabilities and Nonequilibrium Structures, edited by E. Tirapegui and D. Villaroel (Reidel, Dordrecht, 1987), pp. 63-88.

[12] F. Daviaud and J. M. Vince, Phys. Rev. E 48, 4432 (1993).

[13] M. K. Smith and S.H. Davis, J. Fluid Mech. 132, 119 (1983).

[14] D. Villers and J. K. Platten, J. Fluid Mech. 234, 487 (1992); D. Schwabe, U. Möller, J. Schneider, and A. Scharmann, Phys. Fluids 4, 2368 (1992); J. Burguete, N. Mukolobwiez, and F. Daviaud (to be published).

[15] J. F. Mercier and C. Normand, Phys. Fluids 8, 1433 (1996).

[16] J. Lega, B. Janiaud, S. Jucquois, and V. Croquette, Phys. Rev. A 45, 5596 (1992).

[17] V. Croquette and H. Williams, Physica (Amsterdam) 37D, 300 (1989). Thanks to V. Croquette for providing us with his powerful software XVIN.

[18] N. Bekki and K. Nozaki, Phys. Lett. 110A, 133 (1985).

[19] M. van Hecke, Phys. Rev. Lett. 80, 1896 (1998); N. Mukolobwiez, J. Burguete, and F. Daviaud (to be published). 